\title{
Miscellany
}

\section{The stop-watch game of tennis}

\section{Background}

The research was primarily intended to measure the actual time taken to play a tennis match at top level and to compare the results with total match time. The methodological concepts and the framework for registration of data were based on some of the ideas put forward by Pierre Tallbort, Chief Medical Officer of The French Tennis Federation. Dr. Tallbort suggested that tiredness is not necessarily linked to length of match duration but more so to the style of playing which in the final analysis is associated with length of playing time. Apparently, tiredness of a baseline player is different to that of a serve and volley specialist. The baseline players maintain a more constant pace when performing compared with serve and volley players. It is how hard they do what they do on court that measures the relative efficiencies and explains the differences in energy expended. Time is an important factor in this process. The baseline players maintain a constant pace and would breathe continuously and are seldom in strain when moving along the baseline whilst the serve and volley players breathe more rapidly and in shorter intervals of time and consume more energy because they move in quick rushes towards net and back. The physiological differences of these two types of players are similar in workout between sprinters and middle distance runners in track events. In other words, this would imply that physiological differences influence the way players are conditioned as athletes and adopt the ability to style of playing. One is tempted to conclude that youngsters training tennis at top level today have already adopted a style of playing that is compatible with their physiological qualities.

\section{Definitions and methods of registration}

Playing time is defined as the time taken to play a rally measured from the moment a player positions himself to serve until the end of the rally. The measurement of time was recorded with the stop-watch and hence the term "The stop-watch game of tennis"'. The recordings were done in seconds - not split seconds - and the watch was started and stopped manually. Naturally, this method of time taking is associated with errors due to the human coordination factor between hand and eye. Presently, there is no alternative to this method of manual time taking. However, the recorded observations should give a fairly accurate account of the 
Table 1

Stockholm Open (Supreme court) 1991

5 minutes

Australian Open 1992

$61 / 2$

Wimbledon 1992

Stockholm Open (Green Set Trophy surface) 1992

$61 / 2$

Paris Roland Garros (1980-85)

time taken to play a rally and the aggregation of time per set and match. Firstly, the experience of time taking extends now to four major tournaments and this fact can be supportive of the view about experience in handling the task of manual time taking. Secondly, the fact that seconds were recorded and not split seconds should also be supportive to this point of view. It may be possible at some future date to introduce electronic devices for time taking, for example, in combination with the IBM/Sensor recording services.

The stop-watch method of recording playing time was supplemented with measurements of time taken for relaxation in between serves. This was done in order to measure the differences in relaxation time, if any, and to evaluate whether or not there were any significant differences in relaxation time that could be associated with different styles of playing.

A word of caution is necessary, however, because the data recorded were not for purposes of testing a hypothesis or theory but merely to apply statistical reasoning to a set of observations.

\section{Results of time taking}

Playing time was recorded for the duration of each rally. In this way, the data can easily be aggregated to the level of game, set and match. For analytical purposes it is sometimes convenient to present the data in terms of playing time per set. This has been done for the mens singles finals of four major tennis tournaments. The figures of playing time per set are indicated in Table 1.

The recordings were done at court side in Stockholm whilst the data for the Australian and Wimbledon matches were made from TV-transmissions. The playing time (averages) for Paris were recorded from video replays.

The results are revealing: Actual playing time of the finals was of shorter duration on the fast surfaces like the "supreme court" in Stockholm 1991 and extended on the slower clay courts as in Paris. According to these measurements, the actual playing time, that is, the entertainment factor in tennis averages about $20 \%$ of total match duration irrespective of court surface quality. In other words $80 \%$ of time spent during a tennis match were for other movements than playing tennis. In comparison, one should mention that the average playing time of the semi-finals and finals at the World Championships in football in Italy in 1990 was about 50\% of match time. On the other hand, the duration of a tennis match at top level is nearly twice as long as football (170 minutes compared to 90$)$.

In all of these top tennis matches the serve and volley specialists won a major 
Table 2

Number of games played by playing time and winner

\begin{tabular}{|c|c|c|c|c|c|c|}
\hline & Numbe & games won & laying $\mathrm{t}$ & & & \\
\hline & Less th & seconds & $31-60$ & nds & $61 \mathrm{seco}$ & or more \\
\hline & Agassi & Ivanisevic & Agassi & Ivanisevic & Agassi & Ivanisevic \\
\hline Set no. & & & & & & \\
\hline 1 (tie break) & 0 & 3 & 4 & 4 & 3 & 0 \\
\hline 2 & 3 & 1 & 2 & 3 & 1 & 0 \\
\hline 3 & 3 & 3 & 3 & 1 & 0 & 0 \\
\hline 4 & 1 & 3 & 0 & 3 & 0 & 0 \\
\hline 5 & 1 & 4 & 3 & 0 & 2 & 0 \\
\hline Total & 8 & 14 & 12 & 11 & 6 & 0 \\
\hline
\end{tabular}

share of the shorter rallies and the baseliners a major share of the longer rallies. Naturally, some of the players are trained baseline players like Borg, Lendl, Chang, Agassi and Courier who are able to hit the ball to length with considerable accuracy and in doing so to extend playing time. The serve and volley specialists like McEnroe, Becker, Stich, Edberg and Ivanisevic are trained to win rallies either through aces or serves and volleys that do not come back into play (shorter rallies). Therefore, the results as measured by playing time should give a fairly accurate account of the outcome.

The stop-watch results from the mens singles finals at Wimbledon 1992 (Table 2) should give some ideas about the statistical effort in explaining the outcome.

Tie-break in the first set was divided into two games based on who was serving. The playing time of rallies with Agassi serving was 60 seconds and with Ivanisevic serving 50 seconds.

The results: Agassi beat Ivanisevic 3-2 in sets. 14 of 22 games with a playing time of less than 30 seconds were won by Ivanisevic. Both players were equally good at winning the 23 games that lasted 31-60 seconds. However, Agassi was the better of the two in winning games lasting more than 61 seconds.

\section{The results of speed watch}

The stop watch recordings were supplemented with recordings of service delivery speed in order to study its impact on winning rallies. The speed recordings were taken from the IBM/Sensor registration of speed for each serve. The data were compiled for the mens singles finals between Guy Forget and Goran Ivanisevic in Stockholm 1992. Figures 1 and 2 show the distribution of the total of 324 serves by the two players at varying speed ranges. The facts reveal that aces were normally associated with high delivery speed but the aces do not account for more than about $16 \%$ of all serves at top speed level ranging from 170 to $199 \mathrm{~km} / \mathrm{h}$. On the other hand, $84 \%$ of all aces were delivered at top speed. However, further research has to be done before one can actually confirm whether or not the speed factor by 


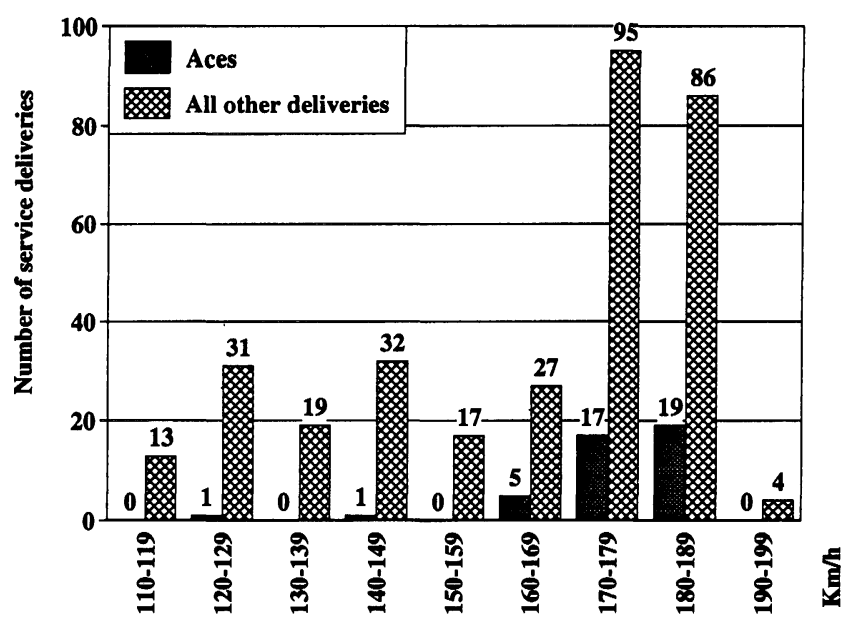

Figure 1. Service delivery by speed $(\mathrm{Km} / \mathrm{h})$ for both players in the Mens Singles Finals Stockholm Open 1992 (Source: Central Statistical Office of Sweden. Reference Brian Wicklin)

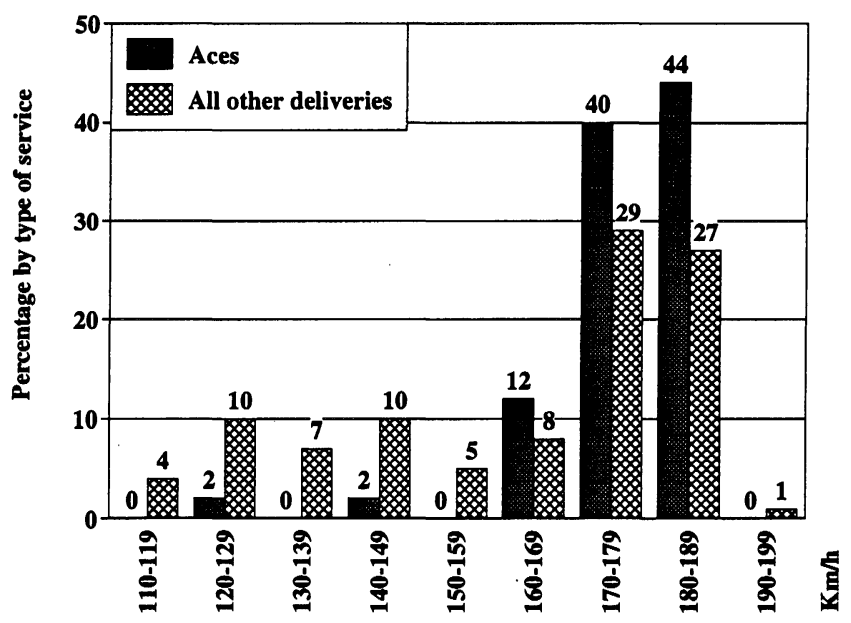

Figure 2. Percentage distribution of aces and other service deliveries by speed $(\mathrm{Km} / \mathrm{h})$ for both players in the Mens Singles Finals Stockholm Open 1992 (Source: Central Statistical Office of Sweden. Reference Brian Wicklin)

itself is sufficient to explain the outcome of a service delivery. For example, $16 \%$ of all aces were delivered at low speed, which suggests that the speed factor is perhaps less important for determining the outcome of a service delivery than is commonly accepted. The area where the ball bounces on the opponent's side is probably more crucial for the outcome than speed. 
Table 3

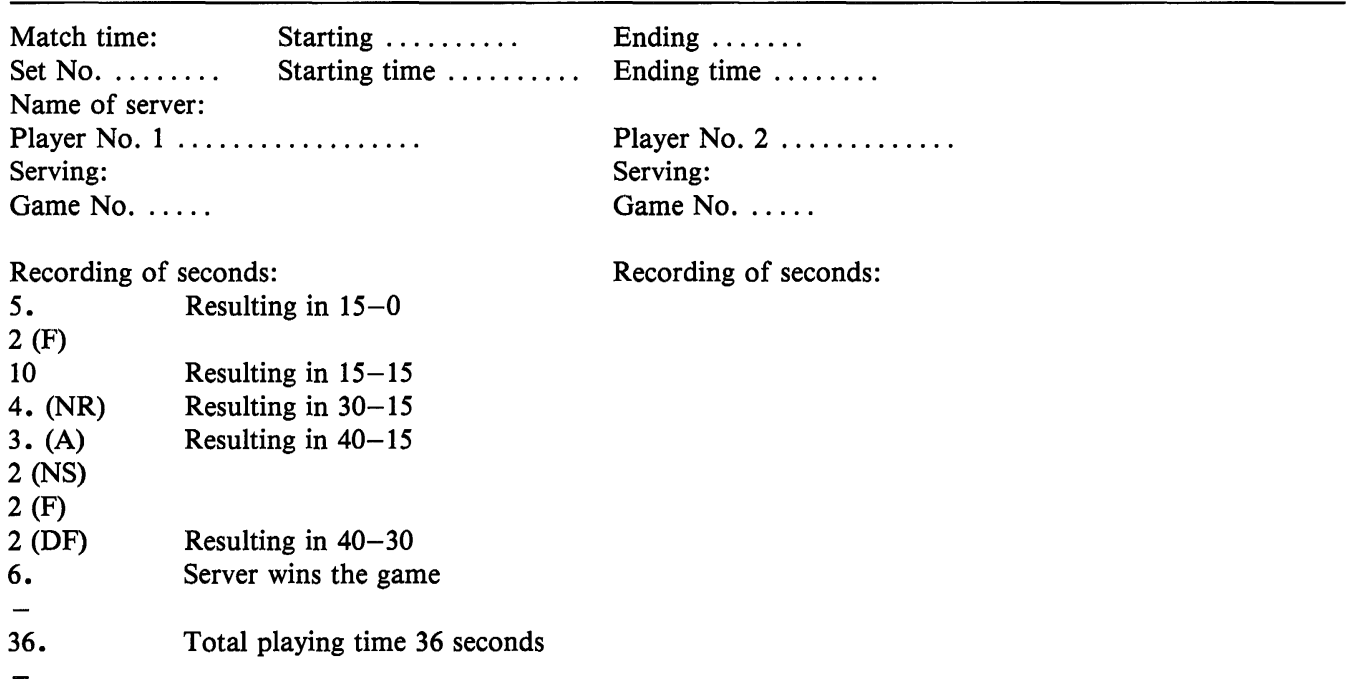

- Server wins the rally/game.

$\mathrm{F}=$ Fault $\quad \mathrm{NR}=$ No return $\quad \mathrm{A}=$ Ace $\quad \mathrm{N}=$ Net service $\quad \mathrm{DF}=$ Double fault

The recording framework (specimen recording) is shown in Table 3. The relaxation time taken in between serves was recorded with the stop-watch technique of time taking.

\author{
Brian Wicklin \\ Statistiska Centralbyrån \\ Sweden
}

